\title{
Lithium Toxicity in an Elderly Women within Normal Therapeutic Range : A Case Report
}

Farhana Mahmood ${ }^{1 *}$

'Department of Medicine Chattagram Maa-O-Shishu Hospital Medical College Chittagong, Bangladesh.
*Correspondence to:

\section{Dr. Farhana Mahmood}

Assisstant Professor

Department of Medicine

Chattagram Maa-O-Shishu Hospital Medical College Chittagong, Bangladesh

Mobile: +8801795199782

Email: farhanadr@gmail.com

www.banglajol.info/index.php/CMOSHMCJ

\begin{abstract}
Toxic effects of lithium are usually related to high serum levels. However, several cases of significant toxicity have occurred in patients whose serum levels fell within the normal therapeutic range. Such cases are rare, but individual variations may occur and clinical judgment rather than serum levels must determine the management. A 67 years old female with bipolar disorder on lithium was admitted with an acute confusional state, tremor, restlessness, increased nausea, slurred speech, polyuria and diarrhoea. Lithium level found to be $0.6 \mathrm{mmol} / \mathrm{L}$ that is in normal range. Neurotoxicity could be suspected at serum lithium levels which are considered therapeutic in young adults.
\end{abstract}

\section{Key words}

Elderly; Lithium toxicity; Neurotoxicity; Cognition.

\section{INTRODUCTION}

Lithium is a potent agent for the control of psychiatric disease. It is used as mood stabilizing drug primarily for bipolar disorder. A number of adverse events especially in elderly is documented. Polypharmacy and co-morbid medical/psychiatric conditions in older adults result in potential drug interactions. Fine tremor of hands, dry mouth, mildly increased thirst, mild polyuria and transient mild nausea is observed as initial response to therapy while dose-related lithium intoxication varies according to blood level. It ranges from toxic blood level of $1.5 \mathrm{mEq} / \mathrm{L}$ to $4 \mathrm{mEq} / \mathrm{L}$. It starts with milder sign and symptom like nausea, vomiting, diarrhea and progresses to life threatening seizure, cardiac arrhythmia, hypotension and peripheral vascular collapse as drug level increases ${ }^{1}$.

\section{CASE REPORT}

A 67 year old female, known case of bipolar disease and hypertension on lithium carbonate, haloperidol, procyclidine and clonazepam admitted with an acute confusional state, restlessness, nausea, polyuria and diarrhea. The laboratory data upon presentation is shown in Table-1. On examination, she was afebrile with pale and dry skin. Blood pressure was 125/85 $\mathrm{mm} \mathrm{Hg}$, pulse 70/min. Electrocardiography was normal. Neurological examination revealed coarse tremor of body and hands. Mental status assessment showed that the patient was anxious, agitated, fidgeting. Though oriented to place and person she looked confused with difficulty focusing and sustaining attention. Patient's restlessness increased with increasing polyuria with $5 \mathrm{~L}$ of urine output over 24 hours. Lithium toxicity suspected though lithium level was within therapeutic range. Lithium stopped upon admission. Fluid replacement was given with normal saline. Patient's conscious level deteriorated after initiation of sodium valporate, risperidone, haloperidol, and clonazepam, which leads to unexplained coma. All antipsychiatric medications were stopped. CT scan and MRI brain was normal at that time. Patient intubated for airway protection and kept in ICU. After 6 days patient regain consciousness and extubated successfully. Patient discharged from hospital after 8 days. 2 weeks later antipsychotic medication started with sodium valporate, risperidone and procyclidine. Patient is doing well at home. 
Table 1 : Laboratory data

\begin{tabular}{lrcr} 
Serum chemistry & 1 $^{\text {st }}$ sample & Subsequent sample & Reference values \\
Sodium (mmol/L) & 132 & 159 & $136-145$ \\
Potassium (mmol/L) & 4.3 & 5.0 & $3.5-5$ \\
Chloride (mmol/L) & 93 & 119 & $96-106$ \\
Bicarbonate (mmol/L) & 25.3 & 24.4 & $25-30$ \\
Creatinine (mg/dl) & 1.3 & 1.2 & M 0.4-1.4, F 0.3-1.1 \\
Urea (mg/dl) & 25 & 52.18 & $10-50$ \\
Serum lithium (mmol/L) & 0.62 & 0.23 & $0.4-1.5$ \\
Glucose (mg/dl) & 115 & 120 & $110-140$ \\
Calcium (mg/dl) & 10.4 & 9.9 & $8.8-10.2$ \\
SGPT(ALT) U/L & 15 & 52 & $5-10$ \\
TSH (microIU/ml) & 0.546 & 0.521 & $0.350-5.00$ \\
pH & 7.35 & 7.43 & $7.35-7.45$ \\
\hline
\end{tabular}

\section{DISCUSSION}

Uses of Lithium in older patients, especially patients with neurologic or cognitive impairments, is limited due to the lack of well-designed, large clinical trials. The suggestions for use in clinical practice are based mostly on data from pharmacokinetic studies and clinical experience in old age psychiatric patients ${ }^{1,2}$. Elderly patients usually have physical and psychiatric co-morbidities, chronic course of illnesses, incomplete therapeutic response. They are more sensitive to side effects and toxicity of psychotropic drugs, the consequences being severe and disabling ${ }^{3,4}$. Generally, aging is characterized by progressive impairment of functional capacities of all system organs, reduction in homeostatic mechanisms, and altered response to receptor stimulation. Significant differences in the response to several drugs in same drug concentrations have been observed in older patients as compared to younger ones ${ }^{5}$. Serum concentrations of Lithium in older patients are associated with reduced volume of distribution and reduced renal clearance in patients with hypertension, congestive heart failure or renal dysfunction. At a lower toxic blood level, i.e. $(1.5-2.0 \mathrm{mEq} / \mathrm{L})$ it causes nausea, vomiting, diaorrhea, polyuria, muscular weakness, drowsiness, increasing confusion, slurred speech while in higher toxic blood level i.e. $(2.5-4.0 \mathrm{mEq} / \mathrm{L})$ it causes myoclonic twitches, restlessness, stupor, coma, seizure, cardiac arrhythmia leading to hypotension ${ }^{1,4}$. In aged patients, brain/peripheral lithium concentration ratio can be increased and this may also increase vulnerability to central nervous system toxicity. Some case reports by Sproule et al. (2000] and Young R.C (2005) indicated that lithium toxicity can occur at moderate blood levels $(0.5-0.8 \mathrm{mEq} / \mathrm{L})^{1,4,5}$. Forester et al. (2008) found in a study of bipolar patients that serum lithium levels did not correlate with brain lithium levels in older patients ${ }^{6}$. In addition, higher brain lithium levels, but not higher serum lithium levels, predicted greater executive dysfunction and somatic symptoms of depression in older subjects. Patient shows signs of attention problem like mild delirium or confusion that could be directed physician for toxic serum level of lithium even though older patients are on lower doses and lower target serum level of lithium ${ }^{6,7,8}$.

\section{CONCLUSION}

Mood stabilizer treatment has numerous acute and long-term adverse events. Narrow therapeutic index remains a major limitation of lithium treatment as it requires close monitoring and identification of neurologic adverse events. Selection of newer mood stabilizers should be based both on efficacy and safety according to risk and benefit ${ }^{9,10}$.

\section{DISCLOSURE}

The author declared no competing interest.

\section{REFERENCES}

1. Sproule BA, Hardy BG, ShulmanKI. Differential pharmacokinetics of lithium in elderly patients. Drugs Aging. 2000 Mar;16(3):165-177.

2. Mohandas E, Rajmohan V. Lithium use in special populations. Indian J Psychiatry. 2007;49(3):211-218.

3. Brown KM, Tracy DK. Lithium: the pharmacodynamic actions of the amazing ion. Ther Adv Psycho-pharmacol. 2013;3(3):163-176.

4. Young RC. Evidence-Based Pharmacological Treatment of Geriatric Bi-polar Disorder. PsychiatrClin N Am.2005;28(4):837-869.

5. Trifirò G, Spina E. Age-related changes in pharmacodynamics: focus on drugs acting on central nervous and cardiovascular systems. CurrDrugMetab. 2011;12(7):611-620.

6. Forester BP, Finn CT, Berlow YA, Wardrop M, Renshaw PE, Moore CM, Brain lithium, N-acetyl aspartate and myo-inositol levels in older adults with bipolar disorder treated with lithium: a lithium-7 and proton magnetic resonance spectroscopy study. Bipolar Disord, 2008;10;(6);691-700.

7. Moore CM, Demopoulos CM,Henry ME, Steingard RJ, ZamvilL,Katic A. et al. Brain to lithium serum ratio and age: an in vivo magnetic resonance spectroscopy study. Am JPsychiatry. 2002;159(7):1240-1242.

8. Busko M. "Normal” Serum Lithium Levels Might be Neurotoxicin Geriatric Bipolar Disorder. Medscape. 2008.

9. Price LH, HeningerGR.Lithium in the treatment of mood dis-orders. N Engl J Med. 1994; 331(9):591-598.

10. Berk M, Berk L. Mood stabilizers and treatment adherence in bipo-lar disorder: addressing adverseevents. Ann Clin Psychiatry. 2003;15(3-4):217-224. 